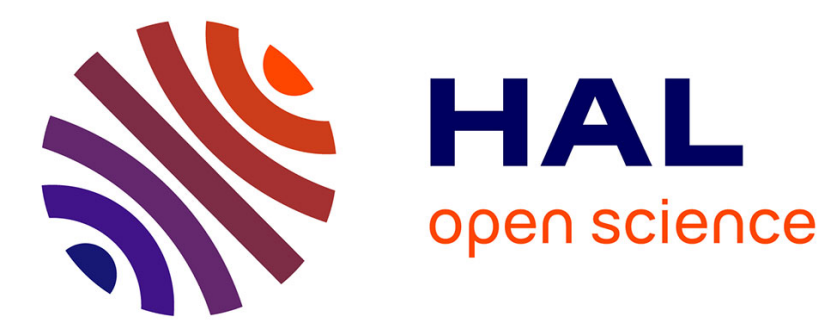

\title{
Quasi-Flat Zones for Angular Data Simplification
}

Erchan Aptoula, Minh-Tan Pham, Sébastien Lefèvre

\section{To cite this version:}

Erchan Aptoula, Minh-Tan Pham, Sébastien Lefèvre. Quasi-Flat Zones for Angular Data Simplification. International Symposium on Mathematical Morphology (ISMM), 2017, Fontainebleau, France. 10.1007/978-3-319-57240-6_28. hal-01672857

\section{HAL Id: hal-01672857 https://hal.science/hal-01672857}

Submitted on 13 Nov 2019

HAL is a multi-disciplinary open access archive for the deposit and dissemination of scientific research documents, whether they are published or not. The documents may come from teaching and research institutions in France or abroad, or from public or private research centers.
L'archive ouverte pluridisciplinaire HAL, est destinée au dépôt et à la diffusion de documents scientifiques de niveau recherche, publiés ou non, émanant des établissements d'enseignement et de recherche français ou étrangers, des laboratoires publics ou privés. 


\title{
Quasi-flat zones for angular data simplification
}

\author{
Erchan Aptoula ${ }^{1}$, Minh-Tan Pham ${ }^{2}$, and Sébastien Lefèvre ${ }^{2}$ \\ 1 Institute of Information Technologies - Gebze Technical University, 41400, Kocaeli, Turkey \\ 2 IRISA - Université Bretagne Sud, UMR 6074, 56000 Vannes, France
}

\begin{abstract}
Quasi-flat zones are based on the constrained connectivity paradigm and they have proved to be effective tools in the context of image simplification and super-pixel creation. When stacked, they form successive levels of the $\alpha$ or $\omega$-tree powerful representations. In this paper we elaborate on their extension to angular data, whose periodicity prevents the direct application of grayscale quasi-flat zone definitions. Specifically we study two approaches in this regard, respectively based on reference angles and angular distance computations. The proposed methods are tested both qualitatively and quantitatively on a variety of angular data, such as hue images, texture orientation fields and optical flow images. The results indicate that quasi-flat zones constitute an effective means of simplifying angular data, and support future work on angular tree-based representations.
\end{abstract}

Keywords: quasi-flat zones, image partition, image segmentation, connectivity, orientation field, hue, optical flow

\section{Introduction}

Although flat zones [1], i.e. connected image regions of constant pixel intensity, represent semantically homogeneous image areas and are thus invaluable for segmentation purposes, they almost always produce oversegmented or too fine image partitions. That is why, there have been multiple attempts at relaxing the pixel connectivity criterion from as early as the 1970s, thus leading to a variety of solutions such as jump connections [2] and quasi-flat zones [3], capable of hierarchical image partitioning and multiscale image representation [4]. Given their potential in terms of image simplification and super-pixel creation, quasi-flat zones in particular have been studied extensively and multiple definitions have been elaborated with varying degrees of flexibility and efficiency [5-8]. Specifically, Soille [7] has provided a solution based on both local and global pixel intensity variation criteria leading to unique image partitions. Angulo and Serra have explored the application of quasi-flat zones in color image segmentation [9], while Aptoula et al. [8] focused on color image simplification. Crespo et al. [10] and Weber et al. [11] have investigated the use of quasi-flat zones in the context of region merging and interactive video segmentation, respectively.

In the light of the success of quasi-flat zones with the simplification and segmentation of grayscale and color images, here we focus on their definition for angular data. In particular, angular images can be encountered in the image processing community in various forms, such as the hue channel of color images in polar color spaces [12], 
oriented textures [13] as well as optical flow datasets [14]. However, the periodicity of angular values most often prevents the direct application of graylevel solutions and instead demands custom definitions. That is why, in this paper we concentrate specifically on the definition of quasi-flat zones for angular data. As a matter of fact, we investigate two approaches, respectively based on angular distances and reference angles. Besides elaborating on their theoretical and practical properties, we also put them to qualitative and quantitative test with the hue channels from the Berkeley dataset, texture orientation fields from Outex database, as well as with optical flow images.

The rest of the paper is organized as follows. Section 2 provides background information on quasi-flat zones, while Section 3 explains the proposed quasi-flat zone definitions for angular data. Experiment results are provided in Section 4, and Section 5 is devoted to concluding remarks.

\section{Background}

Let $f: E \rightarrow T$ be a digital image, where $E$ is its definition domain, the discrete coordinate grid (usually $\mathbb{N}^{2}$ for $2 \mathrm{D}$ images) and $T$ the set of possible pixel values (for instance a subset of $\mathbb{R}$ or $\mathbb{Z}$ ). A path $\pi(p, q)$ between two pixels $p, q \in E$ in this case, is a sequence of $n>1$ pixels $\left(p=p_{1}, \ldots, p_{n}=q\right)$ such that any two successive pixels of the said sequence are adjacent (for instance w.r.t. 4- or 8- adjacency). Moreover, any two pixels are said to be connected if there exists a path between them; while the connected component associated with a pixel $p$ is the set of pixels $C(p)$ containing $p$ and all those connected to $p$. Once one starts taking into account the intensity $f(p) \in T$ associated with each pixel $p$, various custom connectivity relations can be defined, based for instance on pixel-wise intensity dissimilarity (or based on any other pixel attribute, e.g. color purity, texture orientation etc.):

$$
\forall p, q \in E, d(p, q)=\|f(p)-f(q)\|
$$

where $\|\cdot\|$ is a norm. In this case, a couple of pixels $p, q$ are said to be $\alpha$-connected if there exists a path $\pi(p, q)$ between them where the maximal dissimilarity between any couple of successive pixels on the said path is below a certain threshold $\alpha$, thus leading to the definition of $\alpha$-connected components:

$$
\begin{gathered}
C^{\alpha}(p)=\{p\} \cup\{q \mid \widehat{d}(p, q) \leq \alpha\} \\
\widehat{d}(p, q)=\bigwedge_{\pi \in \Pi}\left\{\bigvee_{i \in\left[1, \ldots, N_{\pi}-1\right]}\left\{d\left(p_{i}, p_{i+1}\right) \mid\left\langle p_{i}, p_{i+1}\right\rangle \in \Pi\right\}\right\}
\end{gathered}
$$

with $\Pi$ being the set of all possible paths between $p$ and $q$ and $N_{\pi}$ the length of path $\pi$. Note that flat zones are a particular case of $C^{\alpha}$ where $\alpha=0$. Moreover, a crucial property of $\alpha$-connected components concerns their hierarchy w.r.t. the value of $\alpha$ :

$$
\forall \alpha^{\prime} \leq \alpha, C^{\alpha^{\prime}}(p) \subseteq C^{\alpha}(p)
$$

in other words, for increasing values of $\alpha$, the $\alpha$-connected component associated with a pixel $p$ is guaranteed to contain the previous ones, leading to the so-called $\alpha$-tree representations [15]. This principle has formed the basis of several extensions. As a matter of fact, early examples employing Eq. (2.2) go as back as the late 70s [5,6], followed by various significant extensions and modifications, aiming to remedy inconveniences 
such as the chaining effect and the non-uniqueness of the resulting image partitions. Both of these inconveniences have been resolved by Soille's [7] $C^{\alpha, \omega}$ quasi-flat zones (Fig. 1), where besides $\alpha$, the so-called local variation criterion, an additional global variation criterion $\omega$ is employed:

$$
C^{\alpha, \omega}(p)=\max \left\{C^{\alpha^{\prime}}(p) \mid \alpha^{\prime} \leq \alpha \text { and } R\left(C^{\alpha^{\prime}}(p)\right) \leq \omega\right\}
$$

where $R\left(C^{\alpha}\right)$ is the maximal dissimilarity between the intensities (or some other alternative attribute) of any two pixels of $C^{\alpha}$. Consequently, $C^{\alpha, \omega}$ of a pixel $p$ is the widest $C^{\alpha^{\prime}}$ (i.e. built with the highest $\alpha^{\prime} \leq \alpha$ thanks to property (2.4)) where the maximal inter-pixel dissimilarity is less than or equal to $\omega$. Examples of $C^{\alpha, \omega}$ are shown in Fig. 1. Furthermore, a framework unifying the various quasi-flat zones definitions under the concept of logical predicates has also been proposed by Soille in [16] .

Considering its positive theoretical and practical properties, in the rest of the paper we concentrate exclusively on $C^{\alpha, \omega}$ in order to realize its extension to angular images.

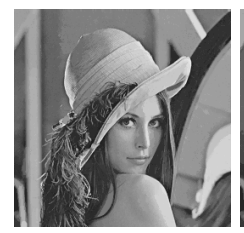

$$
\alpha=\omega=30
$$

$13573 \mathrm{qfz}$

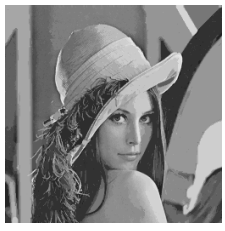

$\alpha=\omega=60$

8344 qfz

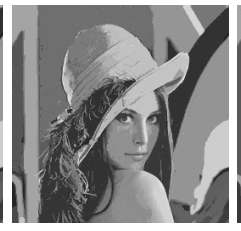

$\alpha=\omega=90$ $5302 \mathrm{qfz}$

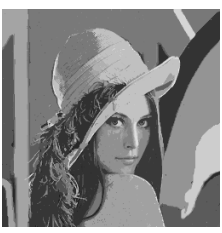

$\alpha=\omega=120$

$4151 \mathrm{qfz}$

Fig. 1: Examples of $C^{\alpha, \omega}$ on $256 \times 256$ Lenna (8-bit) for various local and global criteria.

\section{Angular quasi-flat zones}

The constant diversification of image acquisition means has led to various distinct pixel data types, ranging from scalars (e.g. grayscale images), to vectors (e.g. color, multispectral, hyperspectral images) and even tensors (e.g. diffusion tensor MRI images). Angular images in particular or in other words images where each pixel $p$ represents an angle $f(p) \in[0,2 \pi]$, are often encountered in practice as auxiliary sources of information (Fig. 2). For instance, in the case of color images, polar (or phenomenal) color spaces such as HSV, HLS and their derivatives, describe color in terms of luminance, saturation and hue triplets; the last component is indeed an angular value (Fig. 2a). Other sources of angular content include oriented textures (Fig. 2b), where pixel values represent the local orientation and optical flow images, where each pixel is characterized by a flow vector possessing both a magnitude and an orientation (Fig. 2c).

Although they constitute a rich source of information, the processing of angular images on the other hand is a not a straightforward issue. More precisely, the inherent periodicity of angular data leads to a discontinuity at the origin which very often prevents the direct application of image analysis techniques, that otherwise work perfectly with standard grayscale images, thus rendering it imperative to develop custom solutions adapted to this type of data. 

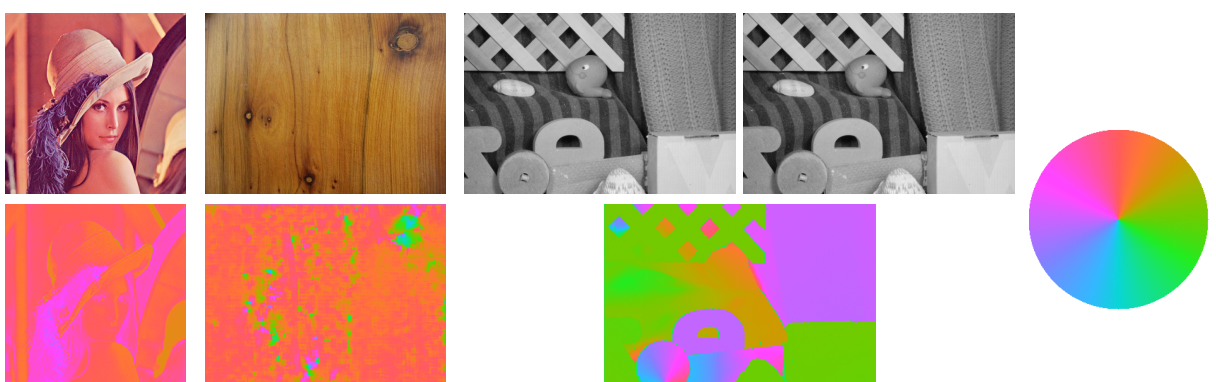

(a)

(b)

(c)

(d)

Fig. 2: Examples of angular images: (a) Lenna color image and (colored) hue channel, (b) wood texture and orientation field, (c) 2 successive frames and the related optical flow orientation, and (d) the color coding used to represent angular data.

Quasi-flat zones are no exception to this situation. Since any two pixels of similar angular value $f(p)=2 \pi-\varepsilon$ and $f(q)=2 \pi+\varepsilon$ located at opposing sides of the origin are bound to be placed in distinct $\alpha$-connected components, thus leading to severe discontinuities. This can be observed in Fig. 3 with the Lenna image, which possesses a mostly reddish hue content (Fig. 3b). Hence, applying $C^{\alpha, \omega}$ directly on its hue channel leads to visually very poor quasi-flat zone results, see Fig. $3 \mathrm{c}$ and Fig. $3 \mathrm{~d}$.

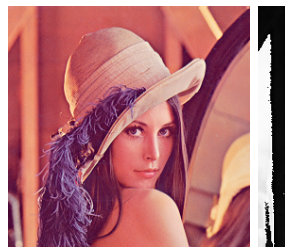

(a) Lenna

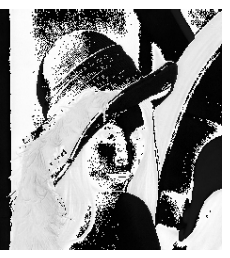

(b) Hue

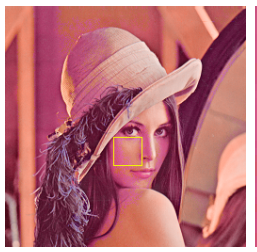

(c) $\alpha=\omega=70$

$$
1803 \mathrm{qfz}
$$

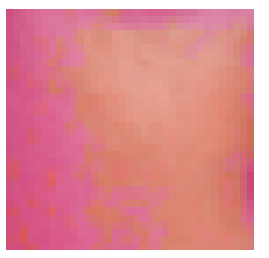

(d) Close-up

Fig. 3: Example of applying $C^{\alpha, \omega}$ directly on the hue channel of a color image, followed by setting each quasi-flat zone to its mean hue.

Given these inconveniences we present in this section two different approaches of computing quasi-flat zones for angular images, that avoid effectively the aforementioned discontinuity problem.

\subsection{Angular distance based approach}

In order to adapt $C^{\alpha, \omega}$ to angular data, both theoretical and practical issues need to be resolved. Let us focus first on the theoretical requirements of this extension. Plus, considering that we now deal with angular images of the type $f: E \rightarrow[0,2 \pi]$, both the local $\alpha$ and global $\omega$ variation criteria represent arc lengths. If one studies carefully Eq. (2.5) of $C^{\alpha, \omega}$, two data dependent parts can be observed that need to be adapted to processing angular data: i) the computation of $C^{\alpha}$ by means of Eq. (2.2), that requires 
the calculation of the dissimilarity $d_{\theta}$ of two angular pixels and its comparison against $\alpha$; ii) and the computation of the maximal dissimilarity $R\left(C^{\alpha}\right)$ between all pixels of $C^{\alpha}$, which once again requires $d_{\theta}$, and its comparison against $\omega$. These are in fact the same requirement: being able to calculate the dissimilarity of any two angles and compare the resulting distance against a predefined distance such as $\alpha$ and $\omega$. However, since the $d_{\theta}:[0,2 \pi]^{2} \rightarrow[0, \pi]$ of any two angles is in fact handled as an arc length, its comparison against $\alpha$ and $\omega$ is trivial; which leaves as sole requirement the definition of $d_{\theta}$. To this end we adopt the solution in Refs. $[12,13]$, where angular distances have been employed as means for establishing a lattice structure on the hue circle of color images:

$$
\forall h, h^{\prime} \in[0,2 \pi], \quad d_{\theta}\left(h, h^{\prime}\right)=\left\{\begin{array}{lll}
\left|h-h^{\prime}\right| & \text { if } & \left|h-h^{\prime}\right|<\pi \\
2 \pi-\left|h-h^{\prime}\right| & \text { if } & \left|h-h^{\prime}\right| \geq \pi
\end{array}\right.
$$

Consequently, we can proceed to define $\alpha$-connected components for angular data $\left(C_{\theta}^{\alpha}\right)$ merely by replacing the distance expression of $C^{\alpha}$ with its angular counterpart:

Thus, we reach the angular $C_{\theta}^{\alpha, \omega}$ :

$$
C_{\theta}^{\alpha}(p)=\{p\} \cup\left\{q \mid \widehat{d_{\theta}}(p, q) \leq \alpha\right\}
$$

$$
C_{\theta}^{\alpha, \omega}(p)=\max \left\{C_{\theta}^{\alpha^{\prime}}(p) \mid \alpha^{\prime} \leq \alpha \text { and } R_{\theta}\left(C_{\theta}^{\alpha^{\prime}}(p)\right) \leq \omega\right\}
$$

where $R_{\theta}$ represents the maximal distance $d_{\theta}$ between any two pixels contained in $C_{\theta}^{\alpha}$. Nevertheless, although $C_{\theta}^{\alpha, \omega}$ is theoretically sound, there are additionally two practical issues that require consideration: i) The amount $\alpha-\alpha^{\prime}$ by which $\alpha$ is going to be decreased each time the global variation criterion is not satisfied, ii) and the computation efficiency of $R_{\theta}$. To explain, when dealing with grayscale images, if the $\alpha \in[0,255]$ argument of $C^{\alpha, \omega}$ leads to an $\alpha$-connected component $C^{\alpha}$ that does not verify the global variation criterion $\omega$, it is the immediately smaller value $\alpha^{\prime}=\alpha-1$ that is employed in its place. With real values however, representing either angular or some other form of data, it is no longer possible to simply select the next smaller value ${ }^{3}$. Consequently, when the initial $\alpha$ value in Eq. (3.3) leads to an $\alpha$-connected component $C_{\theta}^{\alpha}$ that does not verify the global variation criterion $\omega$, a fixed decrementation step $\beta \ll \alpha$ becomes necessary. Thus, if $\alpha$ fails to verify the global criterion, we try next $\alpha^{\prime}=\alpha-\beta$. Naturally, a large $\beta$ value will result in faster quasi-flat zone computations, since less attempts will be made in order to determine the $\alpha^{\prime}$ value that verifies the global criterion, while on the other hand the said $\alpha^{\prime}$ value will be a poorer approximation w.r.t. using a smaller $\beta$ value. A more effective solution for this problem could be to set $\alpha^{\prime}$ to the smallest dissimilarity $d_{\theta, \min }$ between the pixels of $C_{\theta}^{\alpha}$, since the $\alpha^{\prime}$ values such that $d_{\theta, \text { min }} \leq \alpha^{\prime}<\alpha$ will not modify the content of $C_{\theta}^{\alpha}$ and thus have no effect on the global variation criterion.

The second practical issue concerns the computation of $R_{\theta}\left(C_{\theta}^{\alpha}\right)$. To explain, when dealing with standard grayscale images, the calculation of $R\left(C^{\alpha}\right)$ is realized with a linear complexity w.r.t. the number of pixels in $C^{\alpha}$. Each time a pixel is added into $C^{\alpha}$, it suffices to compare it only against the pre-calculated maximum and minimum values, i.e. an operation of constant complexity. However, when it comes to angular images this strategy is no longer applicable, since the new maximal angular distance might be in fact

\footnotetext{
${ }^{3}$ Let us observe however that this issue can be overcome with algorithms specifically designed to build tree-based representations for high-depth data.
} 
between any angle couple and not necessarily between an angle and one of the previous extrema (Fig. 4). The naive approach of calculating $R_{\theta}\left(C_{\theta}^{\alpha}\right)$ would consist in crosscomparing all pixels of $C_{\theta}^{\alpha}$ every time a new pixel is added, which implies a complexity $O\left(n^{3}\right)$ in terms of pixels within $C_{\theta}^{\alpha}$ and is practically unacceptable. An improvement would be to preserve the maximal dissimilarity of $C_{\theta}^{\alpha}$ (a trivial computation for 1 and 2 pixels) and each time a new pixel $q$ arrives into $C_{\theta}^{\alpha}$, one would need to compare only $q$ against the pixels of $C_{\theta}^{\alpha}$ and update the maximal dissimilarity if $q$ possesses a greater distance to $C_{\theta}^{\alpha}$, thus reducing complexity to $O\left(n^{2}\right)$.

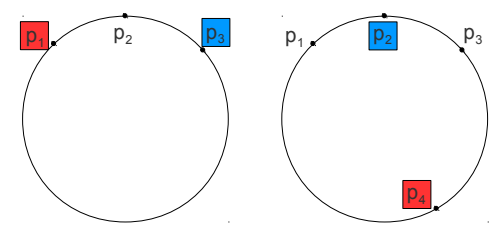

Fig. 4: The most distant angles are illustrated in red \& blue. On the right, it is shown that the most distant couple does not have to contain one of the previous extrema $p_{1}$ and $p_{3}$.

Further acceleration can be achieved if we search for the most distant pixel to $q$ within $C_{\theta}^{\alpha}$ with a binary search. However, in order to avoid the discontinuity at the origin, which prevents the classic application of the binary search algorithm, one can divide the interval $[0,2 \pi]$ into two bins $[0, \pi]$ and $] \pi, 2 \pi]$ and place all pixels of $C_{\theta}^{\alpha}$ into their respective bins, where in each bin they are kept within a sorted data structure. A scalar sort is feasible since the individual bins will not contain the discontinuity at the origin. Next, each time a new pixel $q$ arrives into $C_{\theta}^{\alpha}$, we locate the pixel of maximal distance to $q$ with a binary search within the opposing bin to that of $q$. If the opposing is empty, it suffices to compare against the extreme pixels (that are known thanks to the sorted data structure) of the remaining bin. Of course this is by no means an optimal solution. Besides, as this is the first paper on angular quasi-flat zones, it focuses rather on their feasibility, with computation efficiency being a future work topic.

Fig. 5 shows the quasi-flat zone results obtained for Lenna using various local and global variation criteria. Differences w.r.t. a mere grayscale application (Fig. 3) are emphasized at hue discontinuity regions.

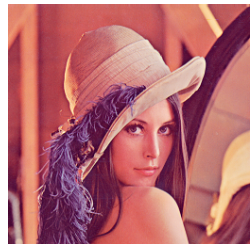

(a) Lenna

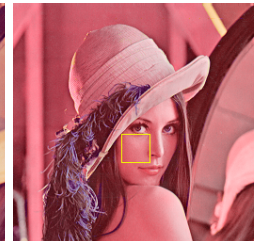

(b) $\alpha=\omega=0.36$ $1896 \mathrm{qfz}$

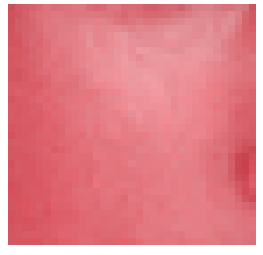

(c) Close-up

Fig. 5: Example of applying $C_{\theta}^{\alpha, \omega}$ (with $\beta=10^{-4}$ ) on Lenna's hue channel by setting each quasi-flat zone to its mean hue. Spatial inconsistencies from Fig. $3 \mathrm{~d}$ have been removed. 


\subsection{Reference angle based approach}

A relatively simpler and more efficient way of computing quasi-flat zones from angular data is to first convert the input into a grayscale image and then apply the efficient algorithm of $C^{\alpha, \omega}$ from Eq. (2.5), as already done with color images [8]. The conversion process can be realized using the angular distance of Eq. (3.1). More precisely, we associate each angular pixel value $f(q) \in[0,2 \pi]$ with its distance $d_{\theta}\left(\theta_{\text {ref }}, f(q)\right)$ to a reference angle $\theta_{\text {ref }} \in[0,2 \pi]$, which leads to a grayscale image of distances. Distance images of Lenna for various reference hues are shown in Fig. 6.

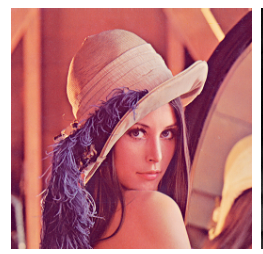

Lenna

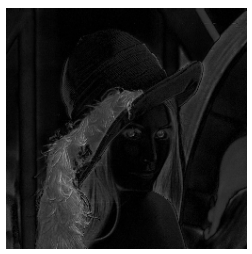

$\theta_{\text {ref }}=0.0$

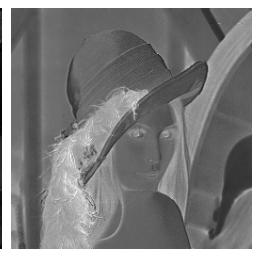

$\theta_{\text {ref }}=0.2$

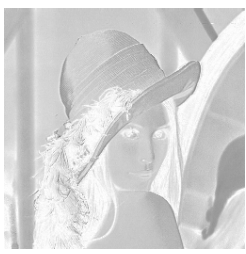

$\theta_{\text {ref }}=0.4$

Fig. 6: Angular distance images of Lenna's hue channel for various reference values.

Once the grayscale distance image is obtained, the computation of quasi-flat zones by means of $C^{\alpha, \omega}$ is straightforward. Fig. 7 shows the quasi-flat zones obtained for $\theta_{\text {ref }}=0.0$. As the results appear visually very similar, if not identical to the previously presented angular distance based approach, one is tempted to adopt the reference based approach for simplicity's sake. Yet, there are serious differences between the two methods. For instance, this one requires not only a reference angle value, but if we are to employ the efficient implementations of $C^{\alpha, \omega}$ then it also subquantizes the resulting pixel values into a range of integers as well, instead of dealing with real-valued angles.

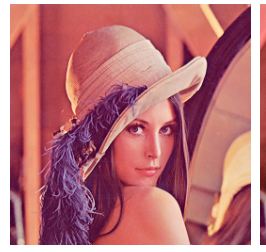

(a) Lenna

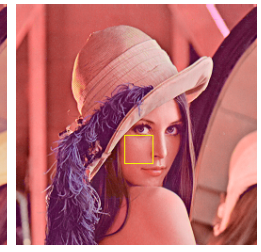

(b) $\alpha=\omega=70$ $3362 \mathrm{qfz}$

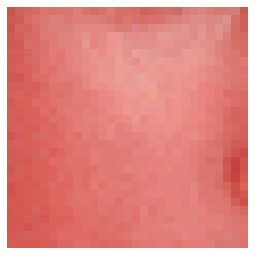

(c) Close-up

Fig. 7: Example of applying $C^{\alpha, \omega}$ on the distance image of Lenna's hue channel using $\theta_{\text {ref }}=$ 0.0 , followed by setting each quasi-flat zone to its mean hue.

Moreover, how can one choose the reference angle? A similar question is encountered during the morphological processing of hue and has been explored in details in [12]. Multiple strategies are available. Since setting it at some arbitrary value can be deemed as a last resort, an alternative is to employ as reference the most dominant angle of the input image, or even better, if multiple dominant angles are present, take them all into account. This is however a direction for future research and we will not focus on it here. In short, concerning the reference angle based approach, the following 
questions arise: What is the effect of the reference angle choice on the quality of the resulting image partitions? Does the performance of the proposed two approaches depend on data type (e.g. hue, orientation field, etc.)? And of course, which one is better suited for image simplification? We now proceed to a series of experiments that will help us answering these questions.

\section{Experiments}

We present here a series of experiments conducted with the main goal of comparing the proposed angular quasi-flat zone approaches against each other and more precisely in order to answer the questions raised at the end of the previous section. As stated in [7], image simplification and super-pixel creation constitute the main applications of quasi-flat zones; that is why they have been chosen for both qualitative and quantitative performance comparison. In particular, we employ three datasets of angular content; for qualitative comparison we use the orientation of the optical flow images made available by Baker et al. [14] and for quantitative image simplification evaluation we use the hue channel of the color images from the Berkeley Segmentation Dataset [17] as well as the orientation fields of the texture segmentation suite Outex-SS-00000 [18].

\subsection{Orientation of optical flow}

The dataset provided in [14] contains the optical flow ground truth of eight images, out of which we picked three for qualitative comparison purposes. We first computed the orientation images of the provided ground-truth and then both angular distance (AQFZ) and reference (RQFZ) based approaches have been computed with distinct $\alpha$ and $\omega$ arguments, so as the final results possess comparable quasi-flat zone numbers. We set $\beta=10^{-4}$ for AQFZ and $\theta_{\text {ref }}=0.0$ for RQFZ. The colored results are shown in Fig. 8, as well as zoomed versions to highlight differences between the two methods occuring at a finer scale.
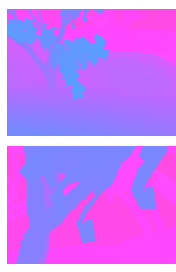

Grove 2
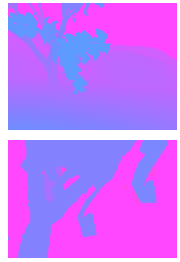

AQFZ (557 qfz) RQFZ (539 qfz)

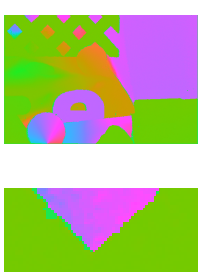

Rubber Whale
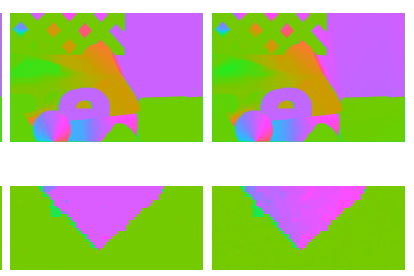

AQFZ (37546 qfz) RQFZ (35838 qfz)

Fig. 8: Colored results (w.r.t. color coding in Fig. 2) of computing angular distance (AQFZ) and reference (RQFZ) based quasi-flat zones from the orientation of optical flow data (top line), and related close-ups (bottom line).

Based on Fig. 8, both AQFZ and RQFZ appear at first to provide results of similar visual quality. However, upon a closer inspection of Grove 2 image, it becomes evident that for a comparable number of quasi-flat zones, RQFZ has a less reliable behavior than 
AQFZ, since it leads to serious under-segmentation. On the hand, we observe RQFZ to preserve a higher level of detail for Rubber Whale image at Fig. 8. As far as AQFZ is concerned, its results appear to be overall spatially and spectrally consistent. We consider the next datasets for a more healthy comparison.

\subsection{Hue channels}

Following the preliminary qualitative evaluation with the orientation of optical flow images, we now focus on a quantitative evaluation of AQFZ and RQFZ through the segmentation of the hue channel of the images contained in the Berkeley dataset [17]. In detail, it contains 300 color images of size $481 \times 321$ pixels and 3269 reference segmentations, realized by 28 distinct experts. The relatively high number of reference segmentation maps per image enables a more objective comparison of our results against real-world practical requirements.

Moreover, in order to evaluate the image partitions produced by either method, two criteria will be employed: the over-segmentation ratio $(O S R)$ [19] and maximal precision $(M P)[20]$. The former is defined as:

$$
O S R=\frac{\text { number of quasi-flat zones }}{\text { number of reference regions }}
$$

This ratio expresses directly the degree of over-segmentation. In fact, in a way it provides us with the merging degree required for achieving the closest possible segmentation to the reference.

$M P$ or maximal precision on the other hand, focuses on pixel-based distances between the reference segmentation and the quasi-flat zones. More precisely, each quasiflat zone is associated with the reference region it shares the highest number of pixels. Consequently, one can compute for each quasi-flat zone the degree with which it overlaps with the desired reference region. Thus, one can calculate a confusion matrix $C$, where $C_{i j}$ represents the number of pixels affected to reference region $i$, while in fact those pixels belong to reference region $j$. Formally:

$$
M P=\frac{\sum_{i=1}^{\text {number of reference regions }} C_{i i}}{\text { number of pixels }}
$$

For instance $M P=0.8$ means that we have achieved $80 \%$ pixel-based accuracy w.r.t. the ground-truth. Hence, by using both $M P$ and $O S R$ one can effectively evaluate a segmentation result, with the ultimate goal being the minimization of $O S R$ and the maximization of $M P$. In order to achieve this goal, we computed both AQFZ $\left(\beta=10^{-4}\right)$ and RQFZ for all the available images, using a wide interval of values for both $\alpha$ and $\omega$ and computed both $M P$ and $O S R$ for all reference segmentation maps, that were then averaged. As far as the reference angle value of RQFZ is concerned, three distinct hues have been employed (red, yellow and green). The resulting plot is shown in Fig. 9a.

Judging from the results, one can assert the overall superiority of AQFZ with respect to RQFZ, both in terms of $M P$ and $O S R$. This difference can be theoretically explained by the fact that AQFZ deals with the actual real-valued angles, thus having access to a higher resolution of $\alpha^{\prime}$ values when searching for the greatest $\alpha^{\prime} \leq \alpha$ that verifies the global variation criterion. While RQFZ on the other hand, transforms its input first into 


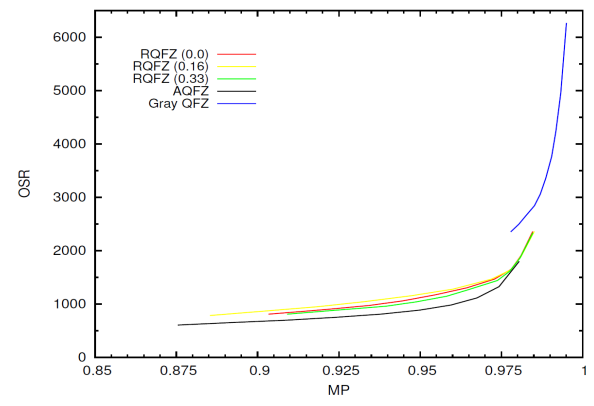

(a) Berkeley dataset

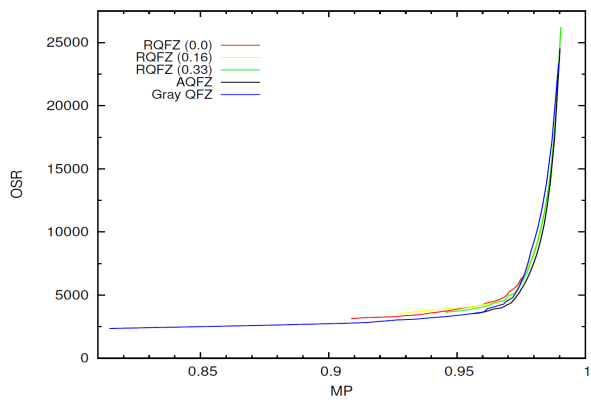

(b) Outex-SS-00000

Fig. 9: $O S R-M P$ plot for the QFZ results of the Berkeley and Outex-SS-00000 dataset. The reference angle in use is indicated between parentheses.

grayscale distance images and thus loses precision prior to realizing grayscale quasi-flat zone computations. Moreover, according to Fig. 9a one can also observe that the choice of reference angle has indeed a non-negligible effect on segmentation performance. The choice of an optimal reference angle however is beyond the scope of this paper.

Furthermore, despite its shortcomings RQFZ has a distinct advantage over AQFZ in terms of execution speed. The average computation time of AQFZ and RQFZ across all images of the Berkeley dataset is $5552 \mathrm{~ms}$ and $400 \mathrm{~ms}$, respectively. Experiments were carried out on a $2.3 \mathrm{GHz}$ system with $3 \mathrm{~GB}$ of memory. Apparently, the relative superiority of AQFZ comes at a very steep computational cost. All the same, as these are the first results on angular quasi-flat zone computation, we are confident that AQFZ can be rendered more efficient, especially if embedded within a tree framework.

\subsection{Texture orientation fields}

As a further source of angular images we have used additionally the orientation field of textures. In particular, the Outex-SS-00000 [18] supervised segmentation test suite contains 100 images, such as the one shown in Fig. 10a, each composed of five distinct textures acquired at various illumination and rotation conditions. Given this grayscale data, for each texture we computed its orientation field according to [21], and subsequently AQFZ and RQFZ similarly as previous experiment. The ground-truth of the segmentation suite being available, the $M P$ and $O S R$ plot that has been obtained is shown in Fig. 9b.

Although the differences are not as emphasized as in the case of hue images, the relative performances are reproduced. Namely, AQFZ is once again superior to RQFZ especially in terms of $M P$ regardless of RQFZ's reference angle, while RQFZ appears once more to be sensitive to the choice of reference angle. The reproduction of the relative performances with two distinct datasets and additionally with distinct image types (hues and orientation fields) constitute a strong indication in favor of AQFZ, even though computationally speaking it is significantly disadvantaged w.r.t. RQFZ. 


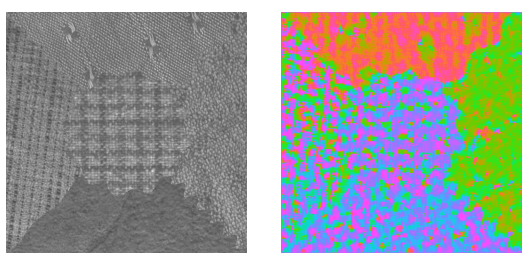

(a) Image 35 of OutexSS (b) Orientation field (colored)

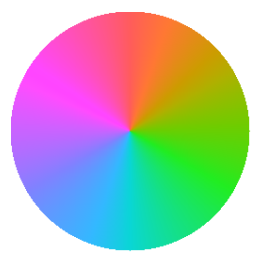

(c) Color coding

Fig. 10: A texture example of OutexSS and its orientation field.

\section{Conclusion}

In this paper, we have adapted the existing definitions of quasi-flat zones to angular data. Both theoretical and practical issues have been addressed. Two distinct approaches have been introduced: angular distance based and reference angle based methods. The former relies on arc lengths for local and global variation comparisons while the latter is based on converting the angular images into grayscale. Following the experimental study using three angular image types, it has been shown that the strong potential of quasi-flat zones as image simplification tools, encompasses angular data as well.

Nevertheless, although the proposed methods can both resolve the discontinuity issues arising when using directly the grayscale methods on angular data, they have significant differences. In detail, the angular distance based approach has been observed to outperform its counterpart with respect to both segmentation criteria that have been employed, while its computational cost and implementation complexity remain much higher. The reference angle based approach on the other hand is simple to implement and efficient, yet it requires setting a reference angle, which is unclear how to realize optimally at this point.

Having established the groundwork for computing quasi-flat zones from angular images, future work will continue on three directions. First and foremost, the angular distance based approach has a serious efficiency problem, which we believe can be resolved with more advanced data structures. Moreover, although the reference angle based method is a fast and relatively effective solution, the need for a reference angle is another issue that needs attention. And last, given the work on color (vectorial) quasiflat zones, the combination of both orientation and magnitude of optical flow pixels during quasi-flat zone computation is another direction worth exploring.

Let us also observe that having brought some first definitions of constrained connectivity on angular data, we can now process such data using multiscale tree based representations, i.e. hierarchies of partitions such as $\alpha$-tree and $\omega$-tree (but also binary partition tree). The availability of efficient algorithms to build such representations from scalar values [22] will certainly ease tackling computational issues raised in this preliminary study, and demonstrate the potential of multiscale representations for angular data. 


\section{References}

1. Salembier, P., Serra, J.: Flat zones filtering, connected operators, and filters by reconstruction. IEEE Trans. Image Proc. 4 (1995) 1153-1160

2. Serra, J.: Connectivity for sets and functions. Fundamenta Informaticae 41 (2000) 147-186

3. Meyer, F.: The levelings. In: Proc. ISMM. (1998)

4. Cousty, J., Laurent, N., Yukiko, K., Silvio, G.: Hierarchical segmentations with graphs: quasi-flat zones, minimum spanning trees, and saliency maps. Tech. report, LIGM (2016)

5. Nagao, M., Matsuyama, T., Ikeda, Y.: Region extraction and shape analysis in aerial photographs. Comp. Graph. Image Proc. 10 (1979) 195-223

6. Hambrusch, S., He, X., Miller, R.: Parallel algorithms for gray-scale digitized picture component labeling on a mesh-connected computer. J. Parallel Dist. Comp. 20 (1994) 56-58

7. Soille, P.: Constrained connectivity for hierarchical image partitioning and simplification. IEEE Trans. PAMI 30 (2008) 1132-1145

8. Aptoula, E., Weber, J., Lefèvre, S.: Vectorial quasi-flat zones for color image simplification. In: Proc. ISMM. (2013)

9. Angulo, J., Serra, J.: Color segmentation by ordered mergings. In: Proc. ICIP. (2003)

10. Crespo, J., Schafer, R., Serra, J., Gratin, C., Meyer, F.: The flat zone approach: a general low-level region merging segmentation method. Signal Process. 62 (1997) 37-60

11. Weber, J., Lefèvre, S., Gançarski, P.: Interactive video segmentation based on quasi-flat zones. In: Proc. ISPA. (2011)

12. Aptoula, E., Lefèvre, S.: On the morphological processing of hue. Image Vis. Comp. 27 (2009) 1394-1401

13. Hanbury, A., Serra, J.: Morphological operators on the unit circle. IEEE Trans on Image Process. 10 (2001) 1842-1850

14. Baker, S., Lewis, D.S.J.P., Roth, S., Black, M., Szeliski, R.: A database and evaluation methodology for optical flow. Int. J. of Comp. Vis. 92 (2011) 1-31

15. Ouzounis, G.K., Soille, P.: Pattern spectra from partition pyramids and hierarchies. In: Proc. ISMM. (2011)

16. Soille, P.: On genuine connectivity relations based on logical predicates. In: Proc. ICIAP. (2007)

17. Martin, D., Fowlkes, C., Tal, D., Malik, J.: A database of human segmented natural images and its application to evaluating segmentation algorithms and measuring ecological statistics. In: Proc. ICCV. (2001)

18. Ojala, T., Mäenpää, T., Pietikäinen, M., Viertola, J., Kyllonen, J., Huovinen, S.: Outex: New framework for empirical evaluation of texture analysis algorithms. In: Proc. ICPR. (2002)

19. Carleer, A., Debeir, O., Wolff, E.: Assessment of very high spatial resolution satellite image segmentations. Photogram. Eng. Remote Sens. 71 (2005) 1285-1294

20. Derivaux, S., Forestier, G., Wemmert, C., Lefèvre, S.: Supervised image segmentation using watershed transform, fuzzy classification and evolutionary computation. Patt. Rec. Lett. 31 (2010) 2364-2374

21. Hanbury, A., Serra, J.: Analysis of oriented textures using mathematical morphology. In: Proc. Workshop Austrian Ass. for Patt. Rec. (2002)

22. Havel, J., Merciol, F., Lefèvre, S.: Efficient tree construction for multiscale image representation and processing. J. Real Time Image Process. (2016) 